\title{
Relations entre les caractères physico-chimiques des laits et leur aptitude fromagère
}

\author{
F Remeuf 1, V Cossin 1, C Dervin 2, J Lenoir 1, R Tomassone 2 \\ 1 INA Paris-Grignon, laboratoire de recherche de la chaire de technologie (INRA), \\ 78850 Thiverval-Grignon; \\ 2 INA Paris-Grignon, laboratoire de biométrie, 16, rue Claude-Bernard, \\ 75231 Paris Cedex 05, France
}

(Reçu le 30 juillet 1990; accepté le 5 avril 1991)

\begin{abstract}
Résumé - Devant les difficultés de fabrication de plus en plus fréquentes, et le souci d'une amélioration des rendements et de la qualité, la profession et la recherche laitières s'attachent à mieux définir les paramètres déterminant la valeur fromagère des laits. L'étude bibliographique montre l'influence primordiale de certains caractères physico-chimiques du lait sur son aptitude à la coagulation par la présure. À partir de 48 échantillons de laits frais individuels, il a été procédé à la détermination de leur composition physico-chimique, de leur aptitude à la coagulation et de leur comportement en microfabrications de types «pâte fraîche» et "pâte pressée». L'analyse statistique des données a permis de confirmer les rôles déterminants des teneurs en caséines et en calcium du lait, et de mettre en évidence les rôles moins connus des proportions relatives des caséines et de certaines caractéristiques de la structure micellaire. Le travail a conduit à l'établissement d'équations qui permettent de prévoir la valeur du rendement fromager.
\end{abstract}

lait de vache / caractère physico-chimique / microfabrication / aptitude fromagère / rendement fromager

Summary - Relationships between physico-chemical characteristics of milks and their cheese-making properties. In view of the increasing difficulties encountered in cheese-making, and with the intention of improving cheese yield and quality, the dairy industry and dairy research are determined to better investigate coagulation mechanism and better define parameters which determine the cheese-making quality of milk. A bibliographic review has been presented which shows the primordial influence on renneting properties of certain physico-chemical characteristics in milk. Forty-eight individual milk samples were taken and their physico-chemical composition, rennet and acid coagulating properties and cheese-making behavior in soft and fresh "micro-cheese-making" determined. Statistical analysis of data confirmed that cheese-making properties are mainly influenced by casein and calcium content, but are also influenced by lesser known factors such as casein proportion and micellar structure. Finally, linear equations have been established for prediction of cheese yield.

cow milk / physico-chemical characteristic / coagulation / cheese-making property / cheese yield 


\section{INTRODUCTION}

Les fromagers observent, depuis plusieurs années, des difficultés de fabrication et une baisse des rendements, ce qui conduit naturellement à s'interroger sur les aptitudes fromagères des laits d'aujourd'hui.

Les programmes de sélection, davantage orientés vers l'augmentation de la production laitière que vers l'accroissement de sa richesse en matière utile, et l'évolution du cheptel bovin, caractérisée par l'extension de la race Frisonne Holstein, se sont traduits par une baisse des teneurs en protéines des laits collectés. Les conditions d'exploitation des troupeaux ont aussi beaucoup évolué au cours des 2 dernières décennies, et il n'est pas certain, surtout depuis la mise en œuvre des quotas, que les vaches laitières reçoivent toujours les rations assurant la sécrétion d'un lait de composition équilibrée. II convient de tenir compte également de la généralisation du refroidissement du lait à la ferme et d'une conservation en cru prolongée qui, avec le développement des bactéries psychrotrophes et l'action des protéases natives ou bactériennes, peuvent être à l'origine de modifications des caséines, préjudiciables au rendement fromager.

Devant une situation qui préoccupe gravement la profession, il était souhaitable que la recherche oriente ses travaux vers une meilleure définition des aptitutdes fromagères des laits. Celles-ci comportent plusieurs aspects : la qualité bactériologique, l'aptitude au développement des bactéries lactiques, la richesse en matière utile, le comportement vis-à-vis de la présure... Sur ce dernier point, on dispose d'un certain nombre de données, mais celles-ci sont parfois divergentes, souvent incomplètes, et divers éléments restent encore insuffisamment connus sur les rapports existant entre la composition des laits et leur comportement en fromagerie.

L'aptitude des laits à la coagulation par la présure représente un volet majeur de leur potentiel fromager. Les laits qui coagulent lentement tendent à former des gels mous, d'égouttage difficile, alors que ceux dont la coagulation est rapide donnent des gels fermes, s'égouttant bien, des gels qui seront à l'origine de caillés de texture et d'humidité satisfaisantes pour un bon affinage (Mocquot et al, 1954).

Parmi les caractéristiques originelles des laits qui exercent une influence déterminante sur leur aptitude à la coagulation, il faut citer le $\mathrm{pH}$, la concentration en caséines et les teneurs en calcium (Brulé et Lenoir, 1987; Lenoir et Schneid, 1987).

Le $\mathrm{pH}$ influence le temps de coagulation, la vitesse de raffermissement du gel et sa fermeté maximale : la coagulation est plus rapide, le gel se raffermit plus vite et sa fermeté est plus grande lorsque le $\mathrm{pH}$ s'abaisse dans l'intervalle 6,7-6,0 (Ramet et Weber, 1980). L'incidence du pH originel sur le comportement du lait vis-à-vis de la présure a été bien mise en évidence dans plusieurs études récentes portant sur des laits individuels ou des laits de troupeau (eg Storry et al, 1983; Grandison et al, 1984a, b; Okigbo et al, 1985a, b).

La concentration du lait en caséines, dans les limites des variations normales, n'a pas d'incidence sur le temps de coagulation (Storry et Ford, 1982); en revanche, elle constitue l'un des paramètres déterminants de la fermeté du gel. Ainsi, une forte corrélation positive a été observée entre la teneur en caséines et la force du gel (Grandison et al, 1984a, b); par ailleurs, il est bien établi qu'un enrichissement du lait en protéines par ultrafiltration, addition de poudre de lait ou de caséinate se traduit par un accroissement marqué de la ferme- 
té maximale (Amram et al, 1982; Storry et Ford, 1982; Mietton, 1986).

L'influence de la teneur en calcium est depuis longtemps reconnue; elle se manifeste sur le temps de coagulation et sur la fermeté du gel. Ainsi, Mocquot et al (1954) ont établi que le rapport $\mathrm{Ca} / \mathrm{N}$ est sensiblement différent dans les laits «lents" et les laits "normaux" ou "rapides" et, selon Flueler (1978), la vitesse de coagulation augmente lorsque le rapport calcium/ phosphate + citrate de la phase aqueuse croît. Par ailleurs, une corrélation assez étroite a été relevée entre la force du gel et la teneur en calcium (Grandison et al, $1984 a, b)$. On sait également que l'addition au lait de chlorure de calcium, une pratique courante en fromagerie, a pour effet de réduire le temps de coagulation et d'accroître la fermeté du gel, cette influence résultant à la fois de l'augmentation de la teneur en calcium ionique, de l'abaissement du $\mathrm{pH}$ et de l'augmentation de la teneur en calcium micellaire, un facteur déterminant de l'aptitude du lait à la coagulation par la présure (Pyne et Mc Gann, 1962; Flueler, 1978).

L'influence d'autres paramètres relevant des caractères originels est moins bien démontrée.

Ainsi, en ce qui concerne les dimensions des micelles, il a été longtemps admis que le temps de coagulation était plus long et la fermeté du gel moindre lorsque le diamètre moyen des micelles était faible (eg Kosikowski et Mocquot, 1958). Les études récentes tendent à remettre en cause cette relation et il semble, au contraire, que les petites micelles soient à l'origine d'un temps de coagulation plus court et de la formation d'un gel plus ferme que les grosses (Waagner-Nielsen et al, 1982; Niki et Arima, 1984; Chahed, 1985).

L'incidence de la composition des caséines n'est pas non plus clairement éta- blie. Daprès les travaux de Storry et al (1983) et de Grandison et al (1984a, b; 1985), il existe une corrélation assez étroite entre le temps de coagulation et la concentration en caséine $\beta$, alors que la fermeté du gel serait plus étroitement liée à la concentration en caséines $\alpha_{s}$ qu'à celle en caséines $\beta$ ou $\kappa$. Ces résultats sont toutefois en contradiction avec ceux de Yun et al (1982) et de Pearse et al (1986), selon lesquels la teneur en caséine $\beta$ détermine plus particulièrement la fermeté du gel. II convient toutefois d'observer que les corrélations existant entre les concentrations des diverses fractions caséiniques et la teneur en caséine totale peuvent masquer la signification réelle des rapports entre la composition des caséines et l'aptitude du lait à la coagulation.

Ces dernières années, plusieurs équipes ont mis en évidence les relations entre la nature des variants génétiques des protéines et le comportement du lait vis-à-vis de la présure (Grosclaude, 1988). II a été notamment observé que les laits contenant le variant $B$ de la caséine $\beta$ ont un temps de coagulation plus court (EI Negoumy, 1972) et donnent des gels plus fermes (Losi et al, 1973) que ceux contenant les formes $A$, mais ce dernier point n'est pas confirmé dans des études plus récentes (eg Marziali et $\mathrm{Ng}$-Kwai-Hang, 1986b). En revanche, la plupart des auteurs s'accordent pour reconnaître que le variant $B$ de la caséine $\kappa$ est associé à une fermeté de gel plus grande que le variant A (Tervala et al, 1985; Marziali et Ng-KwaiHang, 1986b), et cet effet se fait sentir même à teneurs en caséines égales (Ménard et Tusseau, 1986). Toutefois, il convient de relever l'existence d'une liaison génétique étroite entre les locus de structure des caséines, qui se traduit au niveau des populations par une association non aléatoire des allèles de ces locus, appelée haplotype, et ce phénomène rend 
difficile l'interprétation de l'effet d'un seul variant (Grosclaude, 1988).

L'incidence des caractères rhéologiques du gel présure, notamment de sa fermeté, sur les rendements, est généralement reconnue par les fromagers, mais elle n'a pas toujours été vérifiée en fabrications expérimentales. Ainsi, plusieurs études relèvent une augmentation des pourcentages de récupération des caséines et de la matière grasse lorsque la fermeté du gel augmente au décaillage (eg Lombard, 1982; Okigbo et al, 1984; Mietton, 1986). En revanche, Banks et Muir (1984) n'observent pas de différences de rendement en fonction de la fermeté du coagulum.

Compte tenu de ces incertitudes, il nous a paru utile de chercher à préciser les relations entre les caractères physicochimiques originels des laits, leur aptitude à la coagulation et leur comportement en microfabrications fromagères. Pour satisfaire cet objectif, le travail a comporté la réalisation, à partir de laits individuels n'ayant subi aucune modification notable, et sur un nombre suffisant d'échantillons, de 3 types de déterminations :

- une analyse physico-chimique précisant notamment la composition protéique et minérale, les caractéristiques de la structure micellaire et l'identité des génotypes des protéines;
- l'appréciation des aptitudes à la coagulation par la présure et à la coagulation acide par mesures rhéologiques;

- une appréciation du comportement en microfabrications de types "pâte pressée" et "pâte fraîche", par détermination des rendements.

A partir des données acquises, une analyse statistique a permis d'établir les relations entre les variables physicochimiques et technologiques, et de préciser les poids relatifs des différents paramètres sur le comportement fromager.

\section{MATÉRIEL ET MÉTHODES}

\section{Dispositif expérimental - échantillonnage et prélèvements}

Afin de disposer d'échantillons présentant un maximum de variabilité de composition, 48 animaux du troupeau de Frisonnes Holstein de la ferme expérimentale de l'INA-Paris-Grignon ont été répartis dans le dispositif représenté tableau I à raison de 4 animaux pour chacune des 12 combinaisons des niveaux de 3 facteurs : taux de protéines, type d'alimentation, stade de lactation.

L'échantillon de lait analysé est un mélange de la traite complète du soir (conservée à $10^{\circ} \mathrm{C}$ pendant environ $16 \mathrm{~h}$ ), et de la traite complète du matin.

Tableau I. Dispositif expérimental.

Expérimental framework.

\begin{tabular}{|c|c|c|c|}
\hline Facteur & Nombre de niveaux & & Signification des niveaux \\
\hline Taux protéique & 2 & Bas & $<30 \mathrm{~g} / \mathrm{l} ;$ haut $>30 \mathrm{~g} / \mathrm{l}$ \\
\hline Type d'alimentation & 2 & $\begin{array}{l}\text { Hiver } \\
\text { Été }\end{array}$ & $\begin{array}{l}\text { (apport de pulpes et de drèches) } \\
\text { (apport de fourrage vert) }\end{array}$ \\
\hline Stade de lactation & 3 & & Début, milieu, fin \\
\hline
\end{tabular}


Une partie de ce mélange est écrémée à $45^{\circ} \mathrm{C}$; on ajoute un conservateur à la part de lait écrémé servant aux analyses physicochimiques : l'azothydrate de sodium, à la dose de $0,4 \%$.

Un contrôle de la qualité hygiénique des prélèvements est systématiquement réalisé par dépistage des mammites, par la méthode CMT avec le Leucotest Rhône-Mérieux.

\section{Détermination des caractères physico-chimiques}

L'analyse physico-chimique a comporté les déterminations suivantes effectuées en double:

\section{Le fractionnement et le dosage des matières azotées}

Les dosages des protéines, des caséines, des protéines sériques et de la caséine soluble ont été effectués dans les conditions précisées par Remeuf et al (1989).

Les proportions relatives des caséines $\alpha_{s}, \beta$ et $\kappa$, ont été déterminées par FPLC, selon la technique mise au point par Guillou et al (1987). L'identité des variants génétiques des caséines et de la $\beta$-lactoglobuline a été établie selon les protocoles décrits par Wake et Baldwin (1961), Peterson (1963), et Peterson et Kopfler (1966).

\section{Le dosage des matières salines}

Les différentes formes de calcium ( $\mathrm{Ca}$ total, $\mathrm{Ca}$ soluble et $\mathrm{Ca}$ ionisé) et de phosphore inorganique ( $\mathrm{Pi}$ total et $\mathrm{Pi}$ soluble) ont été dosées dans les conditions décrites par Remeuf et al (1989).

Le citrate soluble a été dosé selon la méthode préconisée par Pierre et Brulé (1983).

\section{La détermination des caractéristiques micellaires}

Les dimensions des micelles ont été déterminées à l'aide de l'analyseur de particules Coultronics N4, par mesure du coefficient de diffusion et calcul d'après la loi de Stokes-Einstein
(Holt et al, 1975) dans les conditions opératoires précisées par Remeuf et al (1989).

Le degré d'hydratation des micelles a été estimé selon la méthode de Thompson et al (1969), par mesure de l'humidité du culot d'ultracentrifugation à $80000 \mathrm{~g}$ pendant $1 \mathrm{~h}$ à $20^{\circ} \mathrm{C}$.

\section{Le pH}

Le $\mathrm{pH}$ est mesuré par potentiométrie, à l'aide d'un appareil Metrohm 654.

\section{Détermination des aptitudes à la coagulation et à l'égouttage}

\section{L'aptitude à la coagulation par la présure}

Elle a été appréciée par la mesure du temps de coagulation, selon la technique de Berridge (1952) et par la détermination des caractères rhéologiques du gel, vitesse de raffermissement et fermeté maximale, à l'aide d'un viscosimètre de torsion de Plint (Burnett et Scott-Blair, 1963) dans une version automatisée, selon le modèle préconisé par Gervais et Vermeire (1983).

L'aptitude à l'égouttage du gel présure est déterminée par mesures de la teneur en eau du caillé centrifugé et du poids de sérum séparé par centrifugation.

Ces déterminations sont effectuées au $\mathrm{pH}$ originel des laits et à la température de $30^{\circ} \mathrm{C}$, avec une dose de présure de $25 \mathrm{ml} / 100$ l (présure Boll à $520 \mathrm{mg} / \mathrm{l}$ de chymosine). Les modalités adoptées sont celles décrites par Remeuf et al (1989).

\section{L'aptitude à la coagulation acide}

Elle a été déterminée par appréciation du comportement rhéologique du gel lactique, à l'aide d'un viscosimètre rotatif Contraves type Rhéomat 115, appareil qui permet d'établir le "rhéogramme" caractéristique du comportement rhéologique de la substance, c'est-à-dire la courbe de la tension de cisaillement en fonction de la vitesse de cisaillement dans la gamme des vitesses de $25 \mathrm{~s}^{-1}$ à $3710 \mathrm{~s}^{-1}$. 
On ajoute $30 \mathrm{mg}$ de levain lyophilisé (Eurozyme type MA101) et $0,3 \mathrm{ml}$ de présure liquide (présure Boll à $520 \mathrm{mg} /$ ), diluée au $1 / 10$ au lait $(1 \mathrm{~kg})$ porté à la température de $25^{\circ} \mathrm{C}$. Après une incubation de $20 \mathrm{~h}$, le gel obtenu est brassé à vitesse constante élevée durant $1 \mathrm{~min}$ à l'aide d'un mixer. À partir du rhéogramme, on détermine 2 variables :

- l'indice de viscosité, la viscosité correspondant à la vitesse de cisaillement maximale, après stabilisation pendant $5 \mathrm{~min}$;

- l'indice de comportement $n$ déduit de l'équation décrivant le comportement du gel brassé. Cette équation a pour expression : $\tau=K_{\varepsilon^{n}}(\tau=$ tension de cisaillement, $\mathrm{K}=$ indice de consistance; $\dot{\varepsilon}=$ vitesse du cisaillement).

La valeur de $n$ est comprise entre 0 et 1 , la valeur 1 caractérisant un comportement newtonien.

\section{Détermination du comportement en microfabrications fromagères}

Deux types de microfabrications ont été mis au point :

- une microfabrication de type pâte pressée;

- une microfabrication de type pâte fraîche. Le calcul des rendements corrigés a été effectué en prenant comme références des pourcentages de matière sèche, les valeurs 50 et 17 considérées comme représentatives de ces fabrications.

\section{Microfabrication type pâte pressée}

Le protocole de microfabrication adopté est présenté sur la figure 1.

Les microfabrications sont réalisées à l'aide d'une microcuve Guérin de $10 \mathrm{l}$, thermostatée, munie de dispositifs de découpage et de brassage du caillé. Deux essais sont réalisés sur chaque échantillon de lait. Les contrôles effectués sur le lait, le caillé et le lactosérum (fig 1) permettent d'établir les bilans matières de la fabrication (tableau II), notamment le rendement de manipulation $\left(R_{1}\right)$ et le rendement en matière sèche, corrigé pour un extrait sec constant de $50 \mathrm{~g}$ pour $100 \mathrm{~g}\left(R_{2}\right)$ selon Maubois et Mocquot (1967). Une partie aliquote du lactosérum récu- péré après démoulage, est centrifugée à $37000 \mathrm{~g}$ pendant $1 \mathrm{~h}$ à $20^{\circ} \mathrm{C}$. Le poids de matière sèche du culot de centrifugation, représente la perte en fines, exprimée en g de matière sèche de fines par $\mathrm{kg}$ de sérum.

\section{Microfabrication type pâte fraîche}

Le protocole de microfabrication est présenté sur la figure 2 .

Deux essais sont réalisés sur chaque échantillon de lait. Les contrôles effectués sur le lait, le caillé et le lactosérum (fig 2) permettent d'établir les bilans de la fabrication avec le rendement de manipulation $\left(R_{1}\right)$ et le rendement en matière sèche, corrigé pour un extrait sec constant, $17 \mathrm{~g}$ pour $100 \mathrm{~g}\left(R_{2}^{\prime}\right)$ (tableau II).

\section{Traitement statistique des données}

Les données relatives aux 48 échantillons de lait ont été traitées à l'aide du logiciel STATITCF. Les traitements sont essentiellement effectués par l'étude de différents modèles de régression. La sélection des variables a été faite par la technique classique de sélection progressive; généralement, le seuil d'introduction a été fixé à $5 \%$, plus rarement à $10 \%$. Cela signifie que dans tous les modèles, les coefficients de régression partielle sont au moins significatifs au seuil de $10 \%$ et généralement à celui de $5 \%$. Une attention toute particulière a été portée à l'examen des résidus des modèles, afin de déceler des observations suspectes ou trop influentes. (Pour des références plus précises sur cette modélisation, on pourra se reporter à Tomassone et al, 1983 ou à Tomassone, 1987).

(On trouvera, tableau III, la liste des variables mesurées et leurs abréviations).

\section{RÉSULTATS - DISCUSSION}

\section{Répétabilité des résultats}

Les essais de répétabilité relatifs aux méthodes de détermination de la composition 
LAIT (6 kg) Standardisé à $25 \mathrm{~g} / \mathrm{l}$ de matière grasse

Levain mésophile lyophilisé

Eurozyme MA 101 réactivé $19 \mathrm{~h}$ à $10^{\circ} \mathrm{C}$ dans $40 \mathrm{ml}$ de lait stérile (dose : $3 \mathrm{~g} / 100 \mathrm{~kg}$ )

Présure à $520 \mathrm{mg}$ de chymosine/l (dose : $30 \mathrm{ml} / 100 \mathrm{~kg}$ )

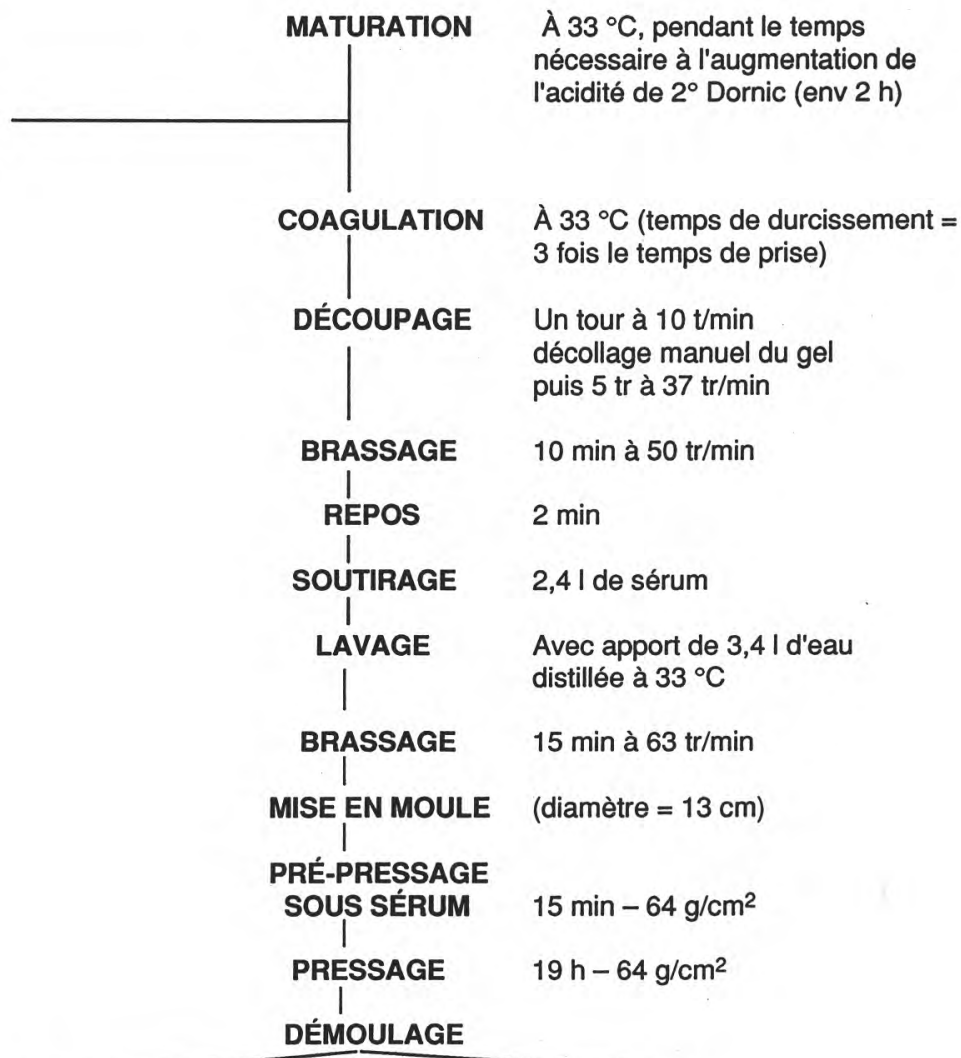

CAILLÉ

Contrôles :

- Poids

$-\mathrm{pH}$

- Extrait sec

- Matières azotées

- Matière grasse

-Calcium total
LACTOSÉRUM

Contrôles :

- Poids

$-\mathrm{pH}$

- Extrait sec

- Matières azotées

- Fines

Fig 1. Protocole de microfabrication type "pâte pressée». Diagram of pressed cheese micro-processing. 


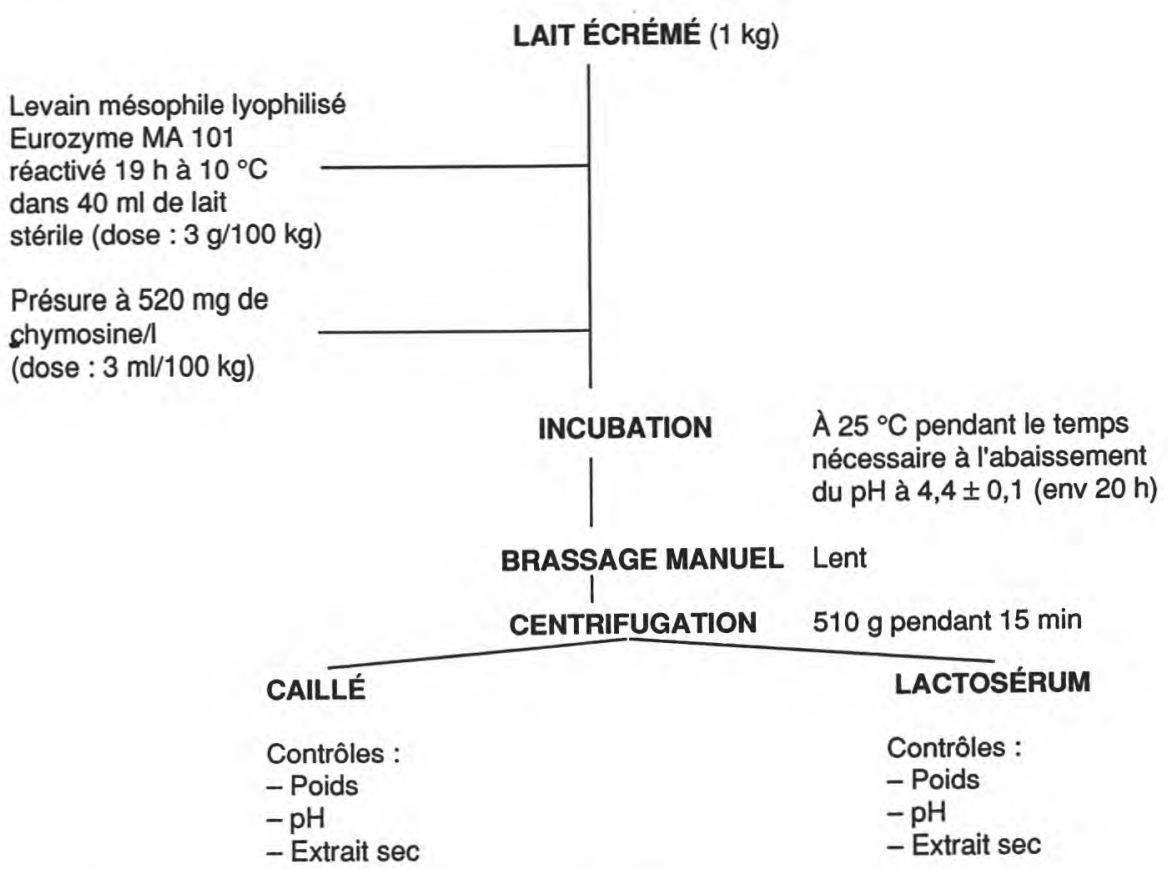

Fig 2. Protocole de micro-fabrication type "pâte fraîche".

Diagram of fresh cheese micro-processing.

physico-chimique des laits ont donné des résultats qui peuvent être considérés comme satisfaisants. Sur 6 déterminations, les coefficients de variation se situent entre 0,8 et $6 \%$. Des coefficients de variation du même ordre de grandeur sont observés pour les méthodes de détermination de l'aptitude à la coagulation.

Des essais de répétabilité ont été également réalisés pour éprouver la fiabilité des microfabrications de types pâte pressée et pâte fraîche. Le tableau IV présente les résultats qui peuvent être considérés comme étant d'une répétabilité convenable.

\section{Variabilité des caractères des échantillons}

\section{Variabilité des caractères physico-chimiques}

Les résultats concernant les caractères physico-chimiques des 48 échantillons sont résumés tableau V. Pour chaque détermination, sont précisés : les valeurs minimale et maximale, la valeur moyenne et le coefficient de variation (noté $\mathrm{Cv}$ et exprimé en \%).

Ces résultats se situent pour la plupart dans les intervalles de variation mention- 
Tableau II. Calcul des rendements des microfabrications.

Calculation of cheese micro-processing yields.

$$
\begin{aligned}
& R_{1}=\frac{\left(P_{c} \times E S_{c}\right)+\left(P_{s} \times E S_{s}\right)}{\left(P_{l} \times E S_{l}\right)} \times 100 \\
& R_{2}=\frac{P_{c} \times 50}{P_{1} \times E S_{l}} \times \frac{E S_{c}-E S_{s}}{50-E S_{s}} \times 100 \\
& R_{2}^{\prime}=\frac{P_{c} \times 17}{P_{1} \times E S_{1}} \times \frac{E S_{c}-E S_{s}}{17-E S_{s}} \times 100 \\
& R_{3}=\frac{100 \times P_{c}}{P_{1} \times N_{1}} \times\left[N_{c}+N_{s} \times\left(\frac{E S_{c}-E S_{s}}{50-E S_{s}}-1\right)\right]
\end{aligned}
$$

$A_{1}$ : rendement de manipulation. $A_{2}$ : rendement en matière sèche de la fabrication de pâte pressée, corrigé pour un extrait sec constant $(50 \mathrm{~g}$ pour $100 \mathrm{~g}) . R_{2}^{\prime}$ : rendement en matière sèche de la fabrication de pâte fraîche, corrigé pour un extrait sec constant (17 g pour $100 \mathrm{~g}) . R_{3}$ : rendement en matières azotées de la fabrication de pâte pressée, corrigé pour un extrait sec constant $(50 \mathrm{~g}$ pour $100 \mathrm{~g}) . P_{1}$ : poids du lait en $\mathrm{g}$. $P_{\mathrm{c}}$ : poids du caillé en $\mathrm{g} . P_{s}$ : poids du lactosérum en $\mathrm{g}$. $E S_{\text {l }}$ : extrait sec du lait en $\mathrm{g}$ pour $100 \mathrm{~g}$. $E S_{c}$ : extrait sec du caillé en g pour $100 \mathrm{~g}$. $E S_{S}$ : extrait sec du lactosérum en g pour $100 \mathrm{~g} . N_{1}$ : teneur en azote du lait en $\mathrm{g}$ pour $100 \mathrm{~g} . N_{c}$ : teneur en azote du caillé en $\mathrm{g}$ pour $100 \mathrm{~g} . N_{s}$ : teneur en azote du sérum en g pour $100 \mathrm{~g}$.

$R_{1}$ : yield of manipulation. $R_{2}$ : yield in $d r y$ matter from production of pressed paste, corrected for a constantly $d y$ extract ( $50 \mathrm{~g}$ for $100 \mathrm{~g}$ ). $\mathrm{R}_{2}^{\prime}$ : yield in dry matter from production of fresh paste, corrected for a constantly dry extract $\left(17 \mathrm{~g}\right.$ for $100 \mathrm{~g}$ ). $\mathrm{R}_{3}$ : yield in nitrogenous matter from production of pressed paste, corrected for a constantly dry extract (50 g for $100 \mathrm{~g}$ ). $\mathrm{P}_{1}$ : weight of milk in g. $\mathrm{P}_{\mathrm{c}}$ : weight of curdled milk in $\mathrm{g} . \mathrm{P}_{\mathrm{s}}$ : weight of wheys in g. $\mathrm{ES}_{1}$ : dry extract of milk in $\mathrm{g}$ for $100 \mathrm{~g}$. $\mathrm{ES}_{\mathrm{c}}$ : dry extract of curdled milk in $\mathrm{g}$ for $100 \mathrm{~g}$. $\mathrm{ES}_{\mathrm{s}}$ : dry extract of wheys in $\mathrm{g}$ for $100 \mathrm{~g}$. $\mathrm{N}_{1}$ : content of nitrogenous matter of milk in $\mathrm{g}$ for $100 \mathrm{~g}$. $\mathrm{N}_{\mathrm{c}}$ : content of nitrogenous matter of curdled milk in $\mathrm{g}$ for $100 \mathrm{~g}$. $\mathrm{N}_{1}$ : content of nitrogenous matter of milk in $\mathrm{g}$ for $100 \mathrm{~g}$. $\mathrm{N}_{\mathrm{s}}$ : content of nitrogenous matter of serum in $\mathrm{g}$ for $100 \mathrm{~g}$.

nés dans la littérature (eg Jenness et Patton, 1959; Alais, 1984). II convient cependant de relever l'exception de 2 échantillons dont les teneurs en protéines sont anormalement faibles $(14,8$ et $19,3 \mathrm{~g} / \mathrm{l})$ et l'intervalle de variation particulièrement large concernant les proportions de caséines solubles.

Si l'on fait abstraction de ce dernier paramètre, on notera que les valeurs des $\mathrm{Cv}$ sont le plus souvent comprises entre 10 et $25 \%$.

Les proportions relatives des fractions caséiniques $\alpha_{s}, \beta$ et $\kappa$, exprimées en $\%$, de la somme $\alpha_{s}+\beta+\kappa$, présentent des valeurs moyennes voisines des données de la littérature (Schmidt, 1980; Swaisgood, 1982).

On relève cependant que la proportion de caséine $\kappa$ est un peu plus faible que celle généralement admise (eg Schmidt, 1980 ) et que son intervalle de variation, d'amplitude 1 à 2 , est particulièrement élevé.

Les fréquences de distribution des variants génétiques des protéines dans les échantillons étudiés concordent avec celles signalées par Swaisgood (1982) pour la race Frisonne-Holstein (tableau VI), avec toutefois un léger écart pour la $\beta$ lactoglobuline.

\section{Variabilité des caractères technologiques}

Les $\mathrm{Cv}$ des paramètres caractéristiques de l'aptitude à la coagulation par la présure sont élevés, compris entre 33 et $50 \%$ (tableau VII); ils permettent ainsi de bien différencier le comportement des laits.

Pour ce qui concerne les microfabrications de type pâte pressée, on peut observer que les paramètres relevant essentiellement de la technologie, $\mathrm{pH}$ et pourcentage d'extrait sec du caillé par exemple, connaissent des valeurs moyennes normales pour ce type de pâte, et des $\mathrm{Cv}$ faibles; en revanche, ceux qui sont influencés par la qualité du lait de fa- 
Tableau III. Liste des variables et des abréviations.

List of variables and abreviations.

Abréviation

Explication

Déterminations effectuées sur le lait et les gels

ANP

Ca ionisé

Ca colloïdal

Ca soluble

Ca total

Caséine $\kappa$

Caséine sol

Caséine $\beta$

Caséines tot

Caséines $\alpha_{s}$

Cit soluble

Diam micelles

Fermeté du gel

Humid caillé centr

Hydr micelles

Indice comportement

Indice viscosité

$\mathrm{Min}^{\circ}$ calcique

Pi colloïdal

Pi soluble

Pi total

Poids sérum égoutté

Protéines

Temps de prise

Variant $\kappa \mathrm{A}$

Variant $\kappa \mathrm{B}$

Variant $\beta$ A2

Variant $\beta$-lacto $A$

Variant $\beta$-lacto $B$

Vitesse de raff du gel
Proportion d'azote non protéique (\% $\mathrm{N}$ total)

Concentration en calcium ionique $(\mathrm{mg} / \mathrm{l})$

Concentration en calcium colloïdal (mg/l)

Concentration en calcium soluble (mg/l)

Concentration en calcium total (mg/l)

Proportion de caséine $\kappa(\%)$

Proportion de caséine soluble (\% caséines tot)

Proportion de caséine $\beta(\%)$

Concentration en caséines totales $(g / l)$

Proportion de caséines $\alpha_{s}(\%)$

Concentration en citrate soluble $(\mathrm{mM} / \mathrm{l})$

Diamètre moyen des micelles $(\mathrm{nm})$

Fermeté maximale du gel présure (unités arbitraires ua)

Humidité du caillé présure centrifugé ( $\mathrm{g}$ eau/100 g MS)

Degré d'hydratation des micelles (g eau/g MS)

Indice de comportement du gel lactique

Indice de viscosité du gel lactique (mPa.s)

Minéralisation calcique des micelles $=\mathrm{Ca}$ colloödal/caséines tot $(\mathrm{mg} / \mathrm{g})$

Concentration en phosphore inorganique colloïdal (mg/l)

Concentration en phosphore inorganique soluble $(\mathrm{mg} / \mathrm{l})$

Concentration en phosphore inorganique total (mg/l)

Poids de sérum égoutté ( $\mathrm{g}$ pour $20 \mathrm{ml}$ de lait)

Concentration en protéines $(\mathrm{g} / \mathrm{l})$

Temps de prise (min)

Variant $A$ de la caséine $\kappa$

Variant $B$ de la caséine $\kappa$

Variant A2 de la caséine $\beta$

Variant $A$ de la $\beta$-lactoglobuline

Variant $B$ de la $\beta$-lactoglobuline

Vitesse de raffermissement du gel présure (ua)

\section{Déterminations effectuées sur le caillé et le lactosérum des microfabrications}

Ca total caillé

Coeff récup MA

Coeff récup MS

ES caillé

Gras/sec

HFD

$\mathrm{pH}$ caillé

Poids fines
Teneur en calcium total du caillé ( $\mathrm{g}$ Ca/100 g MS caillé)

Coefficient de récupération des matières azotées (\%)

Coefficient de récupération de la matière sèche $(\%)$

Extrait sec du caillé (\%)

Teneur en matière grasse du caillé ( $\mathrm{g}$ MG/100 g MS caillé)

Humidité du fromage dégraissé ( $\mathrm{g}$ eau/100 g caillé dégraissé)

$\mathrm{pH}$ du caillé

Pertes en fines dans le sérum (g MS fines $/ \mathrm{kg}$ sérum) 
Tableau IV. Répétabilité des microfabrications ( $\mathrm{Cv}=$ coefficient de variation).

Repeatability of cheese micro-processing ( $\mathrm{Cv}=$ coefficient of variation).

\begin{tabular}{|c|c|c|c|c|}
\hline \multirow[t]{2}{*}{ Variable considérée } & \multicolumn{2}{|c|}{$\begin{array}{c}\text { Pâtes pressées } \\
\qquad(n=6)\end{array}$} & \multicolumn{2}{|c|}{$\begin{array}{l}\text { Pâtes fraîches } \\
\quad(n=12)\end{array}$} \\
\hline & $\begin{array}{l}\text { Valeur } \\
\text { moyenne }\end{array}$ & $\begin{array}{l}\text { Cv } \\
\text { (\%) }\end{array}$ & $\begin{array}{l}\text { Valeur } \\
\text { moyenne }\end{array}$ & $\begin{array}{l}\mathrm{Cv} \\
(\%)\end{array}$ \\
\hline Poids de caillé (g) & 506 & 4,1 & 282 & 2,6 \\
\hline pH du caillé & 5,19 & 0,9 & 4,29 & 0,5 \\
\hline Extrait sec du caillé (\%) & 50,9 & 3,5 & 17,47 & 1,5 \\
\hline Rapport Gras/sec (\%) & 42,4 & 4,1 & $n d^{*}$ & $n d^{*}$ \\
\hline Humidité du fromage dégraissé (\%) & 62,9 & 3,8 & $\mathrm{nd}^{*}$ & $\mathrm{nd}^{*}$ \\
\hline
\end{tabular}

* nd : non déterminé.

* nd: not determined.

Tableau V. Variabilité des caractères physico-chimiques des laits individuels étudiés.

Variability of physico-chemical characteristics in individual milks.

\begin{tabular}{|c|c|c|c|c|}
\hline \multirow{2}{*}{$\begin{array}{l}\text { Variables } \\
\text { Protéines }\end{array}$} & \multicolumn{2}{|c|}{ Valeurs extrêmes } & \multirow{2}{*}{$\begin{array}{c}\text { Moyenne } \\
30,7\end{array}$} & \multirow{2}{*}{$\begin{array}{c}\text { Cv (\%) } \\
13,5\end{array}$} \\
\hline & 19,3 & 38,1 & & \\
\hline Caséines tot & 15,1 & 32,4 & 25,0 & 14,5 \\
\hline Caséine sol & 0,5 & 11,5 & 3,9 & 63,8 \\
\hline ANP & 4,1 & 10,0 & 5,9 & 21,3 \\
\hline Caséines $\alpha_{s}$ & 43,6 & 54,6 & 50,7 & 4,5 \\
\hline Caséine $\beta$ & 35,0 & 47,2 & 39,9 & 6,2 \\
\hline Caséine $\kappa$ & 6,9 & 14,0 & 9,7 & 17,4 \\
\hline Ca total & 942 & 1521 & 1154 & 11,3 \\
\hline Ca colloïdal & 604 & 1062 & 749 & 14,9 \\
\hline Ca soluble & 298 & 632 & 405 & 14,1 \\
\hline Ca ionisé & 63 & 150 & 103 & 18,0 \\
\hline Pi total & 431 & 1209 & 683 & 22,4 \\
\hline Pi colloïdal & 106 & 490 & 259 & 31,5 \\
\hline $\mathrm{Pi}$ soluble & 274 & 799 & 424 & 24,3 \\
\hline Cit soluble & 4,70 & 9,2 & 6,5 & 20,1 \\
\hline Hydr micelles & 1,60 & 2,5 & 2,1 & 10,9 \\
\hline Diam micelles & 142 & 229 & 175 & 11,7 \\
\hline $\mathrm{pH}$ & 6,37 & 6,79 & 6,63 & 1,5 \\
\hline
\end{tabular}


Tableau VI. Fréquence de distribution des variants génétiques des protéines.

Frequencies of protein genetic variants. $\begin{array}{cc}\text { Fréquence } & \text { Race } \\ \text { des variants } & \text { Holstein-Frisonne * }\end{array}$

(\%)

\begin{tabular}{|c|c|c|}
\hline \multicolumn{2}{|l|}{ Caséine $\alpha_{s t}$} & $0-5$ \\
\hline Variant B & 97,9 & $87-99$ \\
\hline Variant C & 2,1 & $1-8$ \\
\hline Variant D & 0 & 0 \\
\hline \multicolumn{3}{|l|}{ Caséine $\beta$} \\
\hline Variant $A 1$ & 58,3 & $31-66$ \\
\hline Variant A2 & 37,5 & $24-62$ \\
\hline Variant $A 3$ & 1 & $1-5$ \\
\hline Variant B & 3,1 & $1-6$ \\
\hline Variant C & 0 & $0-0,1$ \\
\hline Variant D & 0 & 0 \\
\hline \multicolumn{3}{|l|}{ Caséine $\kappa$} \\
\hline Variant A & 75,5 & $75-85$ \\
\hline Variant B & 24,5 & $15-25$ \\
\hline \multicolumn{3}{|c|}{$\beta$-lactoglobuline } \\
\hline Variant A & 41,1 & $46-50$ \\
\hline Variant B & 58,9 & $50-54$ \\
\hline Variant C & 0 & 0 \\
\hline
\end{tabular}

"Référence Swaisgood (1982)

brication, telles les pertes de fines dans le sérum ou le rendement fromager, subissent des variations sensibles.

Les mêmes remarques peuvent être faites au sujet de la variabilité des paramètres de la coagulation acide et de la microfabrication de pâte fraîche. Les $C v$ des caractères rhéologiques du gel et du rendement en matière sèche sont relativement élevés (tableau VIII).

On peut ainsi observer que le plan d'échantillonnage a permis de disposer de laits de composition et de caractères physico-chimiques suffisamment différents pour mettre en évidence des variations si- gnificatives des paramètres caractéristiques de leurs aptitudes fromagères.

\section{Relations entre variables physico-chimiques}

Le tableau IX présente les corrélations les plus significatives concernant les variables physico-chimiques.

La concentration en caséines est fortement corrélée à la teneur en protéines, et le pourcentage de caséines par rapport aux protéines totales, égal en moyenne à $81,5 \%$, varie peu $(\mathrm{CV}=3,9 \%)$. Aussi, les corrélations impliquant la teneur en protéines sont probablement indirectes par rapport à celles qui concernent la teneur en caséines.

Les proportions relatives des différentes caséines ne sont pas indépendantes puisque la somme $\alpha_{s}+\beta+\kappa=100$; ainsi, toutes les interprétations ultérieures doivent se faire en tenant compte de cette contrainte. En revanche, aucune liaison n'est observée entre la teneur en caséines et les proportions relatives des différentes fractions. II n'est pas non plus relevé de corrélation entre la nature des variants génétiques et la concentration en caséines, contrairement aux observations de plusieurs auteurs (Mariani et al, 1976, 1979; Buchberger et al, 1982; Mc Lean et al, 1982, 1984; Ng-Kwai-Hang et al, 1984, 1986; Ménard et Tusseau, 1986). Toutefois, il apparaît que la nature des variants de la caséine $\kappa$ est associée à des variations dans les proportions des fractions caséiniques : le variant $B$ de la caséine $\kappa$ est associé à des laits riches en caséine $\kappa$ $(r=+0,59)$ et pauvres en caséines $\alpha_{s}$ $(r=-0,51)$. Ces résultats, en contradiction apparente avec plusieurs études, peuvent s'expliquer par le mode d'expression des fractions caséiniques, en pourcentage de 
Tableau VII. Variabilité des paramètres de la coagulation présure et de la microfabrication de pâte pressée.

Variability of renneting and pressed cheese micro-processing parameters.

\begin{tabular}{lcccc}
\hline Variables & \multicolumn{2}{c}{ Valeurs extrêmes } & Moyenne & Cv (\%) \\
\hline Temps de prise & 3,50 & 17,8 & 10,2 & 32,2 \\
Vitesse de raff du gel & 0,60 & 5,5 & 2,7 & 42,8 \\
Fermeté du gel & 42 & 179 & 96,7 & 30,1 \\
& 162 & 369 & 241 & 16,8 \\
Humid caillé centrifugé & 16,30 & 18,6 & 17,8 & 3,0 \\
Poids sérum égoutté & 7,1 & 40,0 & 10,6 & 44,3 \\
& 4,97 & 5,25 & 5,12 & 1,6 \\
Poids fines & 44,7 & 51,5 & 48,2 & 3,3 \\
pH caillé & 35,4 & 46,6 & 39,5 & 8,2 \\
ES caillé & 61,5 & 67,7 & 64,1 & 2,2 \\
Gras/sec & 1,2 & 1,8 & 1,5 & 10,8 \\
Humidité du fromage dégraissé & & & & \\
Ca total caillé & 31,9 & 44,9 & 39,7 & 6,9 \\
Coeff récup MS & 58,2 & 81,5 & 69,7 & 8,2 \\
Coeff récup MA & & & & \\
\hline
\end{tabular}

Tableau VIII. Variabilité des paramètres de la coagulation acide et de la microfabrication de pâte fraîche.

Variability of acid coagulation and fresh cheese micro-processing parameters.

\begin{tabular}{lcccc}
\hline Variables & \multicolumn{2}{c}{$\begin{array}{c}\text { Valeurs } \\
\text { extrêmes }\end{array}$} & Moyenne & CV (\%) \\
\hline Indice viscosité & 2,5 & 4,3 & 3,3 & 14,3 \\
$\begin{array}{l}\text { Indice } \\
\text { comportement }\end{array}$ & 0,5 & 1,1 & 0,8 & 17,4 \\
pH caillé & 4,30 & 4,52 & 4,40 & 1,2 \\
ES caillé & 14,7 & 20,6 & 17,7 & 6,5 \\
Coeff récup MS & 32,3 & 49,7 & 41,6 & 9,4 \\
\hline
\end{tabular}

la caséine totale et non en concentration; toutefois, le faible nombre d'observations doit inciter à la prudence.

Parmi les corrélations observées entre les fractions salines, on peut relever celles concernant les concentrations en calcium colloïdal et en phosphore inorganique colloïdal $(r=+0,41)$, les concentrations en calcium soluble et en citrate soluble $(r=$ $+0,50)$. Cette dernière corrélation est en accord avec les observations faites par Holt et Muir (1979).

Les corrélations relatives aux caractéristiques de la structure micellaire sont intéressantes à souligner. Le degré de minéralisation calcique des micelles est corrélé négativement à la concentration en caséines $(r=-0,62)$, mais positivement à la proportion des caséines $\alpha_{s}(r=+0,33)$, fractions qui, du fait de leur richesse en groupements phosphoséryls, ont le pouvoir de fixation de calcium le plus élevé. Le diamètre moyen des micelles est corrélé négativement à la proportion de caséine $\kappa$ $(r=-0,45)$, une liaison déjà bien connue, positivement à celle en caséines $\alpha_{s}$ $(r=+0,51)$. Quant à leur degré d'hydratation, il est lié négativement à la proportion 
Tableau IX. Corrélations entre les variables physico-chimiques.

Correlations between physico-chemical variables.

A : Protéines; B : caséine tot; C : caséine sol; D : caséines $\alpha_{s} ; E$ : caséine $\beta ; F$ : variant kb; $G$ : Hydr micelles; H : Diam micelles; I : Ca colloïdal; J : Min calcique; $\mathrm{K}$ : Ca soluble.

\begin{tabular}{|c|c|c|c|c|c|c|c|c|c|c|c|}
\hline & $A$ & $B$ & C & $D$ & $E$ & $F$ & $G$ & $H$ & 1 & $J$ & $K$ \\
\hline $\begin{array}{l}\text { Caséines tot } \\
\text { ANP } \\
\text { Caséine sol } \\
\text { Caséines } \alpha_{\text {s }} \\
\text { Caséine } \kappa \\
\text { Hydr micelles } \\
\text { Diam micelles } \\
\text { Ca colloïdal } \\
\text { Min calcique } \\
\text { Ca soluble } \\
\mathrm{Pi} \text { colloïdal } \\
\mathrm{Pi} \text { soluble } \\
\mathrm{Cit} \text { soluble } \\
\mathrm{pH}\end{array}$ & $\begin{array}{r}0,96 \\
-0,39 \\
0,38 \\
\\
0,37 \\
0,43 \\
-0,56 \\
\\
-0,37\end{array}$ & $\begin{array}{r}0,41 \\
0,39 \\
0,32 \\
-0,62 \\
-0,30\end{array}$ & $\begin{array}{r}0,45 \\
-0,36 \\
-0,37 \\
0,31\end{array}$ & $\begin{array}{r}-0,46 \\
-0,42 \\
0,51 \\
0,33\end{array}$ & $\begin{array}{l}-0,42 \\
-0,32\end{array}$ & $\begin{array}{r}-0,51 \\
0,59 \\
-0,56\end{array}$ & $\begin{array}{r}-0,34 \\
-0,55 \\
-0,43 \\
0,42\end{array}$ & $\begin{array}{r}-0,45 \\
0,43 \\
0,35\end{array}$ & $\begin{array}{l}0,52 \\
0,41\end{array}$ & 0,38 & 0,50 \\
\hline
\end{tabular}

NB : les coefficients de corrélation indiqués sont significativement différents de 0 au seuil $\alpha=5 \%$

NB : the correlation coefficients indicated differ significantly from 0 at the threshold level of $\alpha=5 \%$.

de caséines $\alpha_{\mathrm{s}}(r=-0,42)$ et à la minéralisation calcique $(r=-0,55)$. Compte tenu des données peu nombreuses dans la littérature concernant les caractéristiques micellaires et leurs incidences majeures au plan de la stabilité du système colloïdal, il est intéressant de rechercher par régression multiple quelles sont les variables physico-chimiques les plus déterminantes (équations [1] et [2]).

Diam micelles $=3,26$. (Caséines $\alpha_{s}$ ) $+0,055$. (Ca colloïdal) - 1,77. (Caséine sol) $-3,55$. (Cit soluble) $-0,74(R=0,67)$

Hydr micelles $=-0,019 .\left(\mathrm{Min}^{\circ}\right.$ calcique $)$ - 0,083. (Cit soluble) + 0,055.(ANP) 0,0029 . (Diam micelles) $+0,0011$. (Ca soluble $)+2,91(R=0,75)$

L'équation de régression du diamètre moyen des micelles [1] montre que les dimensions des particules sont corrélées po- sitivement avec la proportion de caséines $\alpha_{\mathrm{s}}$ et la teneur en calcium colloïdal, et négativement avec la concentration en citrate soluble. Ce sont là des résultats en accord avec les observations de certains auteurs (Niki et Arima, 1984; Donnelly et al, 1984). La corrélation négative observée avec la proportion de caséine soluble est normale, ces deux paramètres traduisant l'état de dispersion de la suspension colloïdale.

L'équation de régression du degré d'hydratation des micelles [2] met en évidence l'influence de la minéralisation calcique, laquelle est à l'origine d'une diminution de la polaritě des caséines, et par suite d'une réduction de leur hydratation. La relation inverse entre la minéralisation des micelles et leur solvatation a été signalée antérieurement par Sood et al (1979).

D'autres variables apparaissent également dans l'équation [2]. La corrélation négative entre le diamètre des micelles et 
leur degré d'hydratation, déjà observée par divers auteurs, peut s'expliquer par la richesse en caséine $\kappa$ et le rapport surface/ volume, les petites micelles plus riches en caséinies $\kappa$ ont un degré d'hydratation superficielle plus élevé.

\section{Relations entre les variables physico-chimiques et les paramètres caractéristiques des aptitudes fromagères en fabrication de pâte pressée}

Le tableau $X$ présente les corrélations significatives entre les variables physicochimiques et les paramètres caractéristiques des aptitudes fromagères en fabrication de pâte pressée.
Le temps de prise paraît être plus particulièrement influencé par le $\mathrm{pH}(r=+0,52)$ et par la teneur en calcium soluble $(r=$ $-0,41)$ et ionique $(r=-0,37)$. La fermeté maximale du gel semble dépendre principalement de la teneur en caséines ( $r=$ $+0,48$ ), des proportions de caséines $\alpha_{s}$ (r $=-0,51)$ et $\beta(r=+0,39)$ et du diamètre moyen des micelles $(r=-0,38)$; la vitesse de raffermissement du gel est notamment influencée par les proportions relatives des caséines $\alpha_{\mathrm{S}}(r=-0,54)$ et $\kappa(r=+0,42)$ et par les dimensions des micelles $(r=-$ $0,46)$. On relèvera que la fermeté du gel et sa vitesse de raffermissement sont plus faibles dans les laits contenant de fortes proportions de caséines $\alpha_{\mathrm{s}}$ et des micelles de dimensions moyennes élevées. Par

Tableau X. Corrélations entre variables physico-chimiques et paramètres de la coagulation présure. Correlations between physico-chemical variables and renneting parameters.

\begin{tabular}{|c|c|c|c|c|c|c|c|}
\hline & $\begin{array}{l}\text { Temps } \\
\text { de } \\
\text { prise }\end{array}$ & $\begin{array}{l}\text { Vitesse } \\
\text { de raff } \\
\text { du gel }\end{array}$ & $\begin{array}{c}\text { Fermeté } \\
\text { du gel }\end{array}$ & $\begin{array}{l}\text { Humid } \\
\text { cailllé } \\
\text { centr }\end{array}$ & $\begin{array}{r}\text { Poids } \\
\text { sérum } \\
\text { égoutté }\end{array}$ & $\begin{array}{l}\text { Coeff } \\
\text { récup } \\
\text { MS }\end{array}$ & $\begin{array}{l}\text { Poids } \\
\text { fines }\end{array}$ \\
\hline Protéines & 0,38 & & 0,44 & & $-0,65$ & 0,67 & 0,54 \\
\hline $\begin{array}{l}\text { Caséines tot } \\
\text { ANP }\end{array}$ & 0,39 & $\begin{array}{r}0,33 \\
-0,30\end{array}$ & 0,48 & & $\begin{array}{r}-0,65 \\
0,32\end{array}$ & 0,67 & 0,45 \\
\hline Caséine sol & & 0,48 & 0,38 & & & 0,45 & \\
\hline Caséines $\alpha_{s}$ & & $-0,54$ & $-0,51$ & & & $-0,52$ & \\
\hline Caséine $\beta$ & & & 0,39 & $-0,35$ & & 0,30 & \\
\hline Caséine $\kappa$ & & 0,42 & & & & & \\
\hline Variant $\beta$ A2 & 0,42 & & & & & & \\
\hline Variant $\kappa \mathrm{A}$ & & & $-0,37$ & & & & \\
\hline Variant $\kappa \mathrm{B}$ & $-0,43$ & 0,50 & 0,40 & & & 0,40 & \\
\hline Variant $\beta$-lacto $B$ & & & 0,30 & & & 0,33 & \\
\hline Diam micelles & & $-0,46$ & $-0,38$ & & & & \\
\hline Hydr micelles & & 0,36 & & & & 0,43 & \\
\hline $\mathrm{Min}^{\circ}$ calcique & $-0,37$ & & & & & $-0,41$ & \\
\hline Ca colloïdal & & & & & $-0,47$ & & 0,34 \\
\hline Ca soluble & $-0,41$ & & & & & & \\
\hline Ca ionisé & $-0,37$ & & & & & & \\
\hline $\mathrm{pH}$ & 0,52 & & & & & & 0,36 \\
\hline
\end{tabular}

NB : les coefficients de corrélation indiqués sont significativement différents de 0 au seuil $\alpha=5 \%$. $N B$ : the correlation coefficients indicated differ significantly from 0 at the threshold level of $\alpha=5 \%$. 
ailleurs, l'incidence favorable du variant $B$ de la caséine $\kappa$ se trouve confirmée.

L'aptitude à l'égouttage, appréciée par le poids de sérum égoutté par centrifugation, est liée plus particulièrement aux teneurs en caséines et en calcium colloïdal. Les corrélations négatives observées ont été mises en évidence dans d'autres travaux (Storry et al, 1983; Remeuf et al, 1989). Elles sont dues probablement au fait qu'un lait riche en caséines contient moins d'eau; aussi, la quantité de lactosérum susceptible d'être libérée à partir d'un volume donné, sera moindre. L'influence du calcium colloïdal s'explique par la liaison positive entre cette variable et la concentration en caséines. Peuvent également intervenir les caractères du gel formé, qui est doté d'une capacité de rétention du sérum d'autant plus grande qu'il est plus ferme.

En ce qui concerne le rendement en microfabrication de pâte pressée, on peut observer que les caractères physicochimiques qui semblent être les plus déterminants sont la teneur en caséines $(r=$ $+0,67)$ et les proportions de caséines $\alpha_{\mathrm{S}}(r$ $=-0,52)$, une faible proportion de caséines $\alpha_{\mathrm{s}}$ étant un facteur améliorant la récupération de la matière sèche.

Le variant $B$ de la caséine $\kappa$ a également un effet positif sur le rendement $(r=$ $+0,40$ ), ce qui rejoint les observations d'autres auteurs (Schaar, 1985; Ménard et Tusseau, 1986; Marziali et Ng-Kwai-Hang, 1986a).

L'analyse par régression multiple permet de mieux apprécier les poids respectifs des différentes variables physicochimiques sur l'aptitude à la coagulation par la présure (équations [3]-[7] et résumé tableau XI).

L'équation de régression du temps de prise [3] confirme l'influence du $\mathrm{pH}$ et de la teneur en calcium soluble; elle met en outre en évidence celle des caractères et de la composition des micelles : l'augmentation du diamètre moyen se traduit par un allongement du temps de prise, et celle de la proportion de caséine $\beta$ ou de la minéralisation calcique, par une réduction.

Temps de prise $=16,2 \cdot \mathrm{pH}+0,054$. (Diam micelles) - 0,452. (Caséine $\beta$ ) $-0,179$. ( Min $^{\circ}$ calcique) $-0,014$. (Ca soluble $)-77,3(R=0,77)$

On notera que la relation entre le diamètre des micelles et le temps de prise a déjà été relevée par divers auteurs (Ekstrand et al, 1981; Waagner-Nielsen et al, 1982; Niki et Arima, 1984; Ford et Grandison, 1986). II en est de même de la corrélation négative (mentionnée tableau $X$ ) entre le temps de prise et le variant $B$ de la caséine $\kappa$ (El-Negoumy, 1972; Mariani et al, 1976; Schaar, 1984, 1985; Ménard et Tusseau, 1986).

L'équation de régression de la vitesse de raffermissement des gels [4] montre que les variables physico-chimiques déterminantes sont les proportions relatives de caséines $\alpha_{s}$, les dimensions et le degré de minéralisation des micelles, le $\mathrm{pH}$. Avec l'augmentation de la proportion de caséines $\alpha_{s}$, il y a diminution de la vitesse de raffermissement du gel, un effet qui pourrait être lié à l'influence de cette variable sur le diamètre moyen des micelles.

Vitesse de raff $=-0,231$. (Caséines $\alpha_{s}$ ) $+0,0037$. (Ca colloïdal) $+0,142$. (Caséine sol) - 0,019.(Diam micelles) - 2,6.pH $+31,4(R=0,76)$

L'incidence de la proportion de caséines solubles est probablement, elle aussi, indirecte; elle peut en effet découler des corrélations avec les dimensions des micelles et (ou) la teneur en caséines (tableau IX), la relation entre la teneur en caséines et la vi- 
Tableau XI. Synthèse des modèles de régression. $R$ : coefficient de détermination ( : niveau $10 \%$, sinon $5 \%$ ); $s$ : écart type de l'échantillor, des observations; $s_{r}$ : écart type résiduel du modèle; $p$ : nombre de régresseurs du modèle; $n$ : nombre d'ob-servations prises en compte.

Summary of regression models. R: determination coefficient (": level $10 \%$ or $5 \%$ ). s: standard deviation of sample of observations. $\mathrm{s}_{\mathrm{r}}$ : standard deviation of residual of the model. $\mathrm{p}$ : number of regression of the model. $\mathrm{n}$ : number of observations.

\begin{tabular}{rlccccc}
\hline $\begin{array}{c}N^{\circ} \\
\text { Equation }\end{array}$ & $\begin{array}{l}\text { Variable } \\
\text { expliquée }\end{array}$ & $s$ & $s r$ & $R$ & $p$ & $n$ \\
\hline 1 & Diam micelles & 19,4 & 15,1 & $0,67^{*}$ & 4 & 43 \\
2 & Hydr micelles & 0,224 & 0,158 & 0,75 & 5 & 43 \\
3 & Temps de prise & 3,28 & 2,21 & 0,77 & 5 & 45 \\
4 & Vitesse de raff du gel & 1,34 & 0,93 & 0,76 & 5 & 43 \\
5 & Fermeté du gel & 32,1 & 20,4 & 0,80 & 4 & 46 \\
9 & Indice viscosité & 0,44 & 0,24 & 0,85 & 1 & 38 \\
10 & Indice comportement & 0,147 & 0,104 & $0,75^{*}$ & 4 & 36 \\
6 & Coeff & 2,78 & 1,64 & 0,83 & 4 & 44 \\
8 & récup & 2,76 & 1,70 & 0,79 & 1 & 45 \\
11 & MS & 3,82 & 1,72 & 0,90 & 2 & 40 \\
12 & & 3,88 & 2,01 & 0,86 & 2 & 38 \\
7 & Coeff récup MA & 5,69 & 4,04 & 0,74 & 4 & 42 \\
\hline
\end{tabular}

tesse de raffermissement du gel ayant été souvent observée (Storry et Ford, 1982; Okigbo et al, 1985a; Tervala et al, 1985; Marziali et Ng-Kwai-Hang, 1986b). Quant aux effets favorables de l'accroissement de la concentration en calcium colloïdal et de la diminution du $\mathrm{pH}$, ils sont déjà bien connus.

L'équation de régression de la fermeté maximale du gel [5] confirme l'influence dominante de la teneur en caséines et celle des dimensions des micelles, déjà relevées dans le tableau X. II apparaît ainsi bien établi que les laits contenant de petites micelles donnent des gels plus fermes; un résultat qui est en accord avec les observations de plusieurs études récentes (Niki et Arima, 1984; Chahed, 1985; Ford et Grandison, 1986).

Fermeté du gel $=6,32$. (Caséines tot) $-0,75$. (Diam micelles) $+3,00$. Min $^{\circ}$ calcique $)+5,04$. (Caséine $\beta)-224(R=0,8)[5]$

La liaison positive entre la fermeté du gel et la minéralisation calcique des mi- celles est connue (Jen et Ashworth, 1970; Lombard, 1982; Storry et al, 1983; Grandison et al, 1984b; Mc Mahon et al, 1984; Okigbo et al, 1985c). Quant à la liaison partielle positive entre la fermeté du gel et la proportion de caséine $\beta$, elle peut paraître en contradiction avec certains travaux (eg Marziali et $\mathrm{Ng}$-Kwai-Hang, $1986 b)$ qui ont noté une augmentation de la fermeté du gel avec l'augmentation de la concentration en caséines $\alpha_{\mathrm{s}}$, mais dans le cas présent, le modèle intègre 4 variables, et le mode d'expression des caséines en pourcentage relatif est différent de celui adopté dans les travaux précités. D'ailleurs, d'autres auteurs ont également mis en évidence l'influence positive de la caséine $\beta$ sur les caractères rhéologiques du gel présure (Yun et al, 1982; Pearse et al, 1986). Celle-ci pourrait s'expliquer par le rôle majeur attribué à la caséine $\beta$ dans la fixation du phosphate de calcium colloïdal et la structuration de la micelle (Rose, 1969), un rôle que suggère le parallélisme observé lors du refroidissement du lait 
dans la solubilisation de la caséine $\beta$ et la migration du phosphate de calcium micellaire (Pierre et Brulé, 1981).

On peut cependant se demander si, dans la présente étude, la moins bonne aptitude technologique des laits à faible teneur en caséine $\beta$ n'est pas la conséquence d'une dégradation partielle de celle-ci, par action de la plasmine. Bien que nos analyses ne permettent pas de vérifier ce point, il convient de souligner que les échantillons de lait ont été prélevés et traités dans des conditions limitant au maximum les altérations enzymatiques. L'absence de différence significative dans la proportion de caséine $\beta$ entre les laits de début, de milieu et de fin de lactation, dont les activités plasmine peuvent être différentes conforte l'hypothèse d'une faible incidence de la protéolyse.

L'équation de régression, relative au coefficient de récupération de la matière sèche [6] montre que celui-ci semble être plus particulièrement influencé par les paramètres qui déterminent l'aptitude à la coagulation par la présure : la teneur en caséines, les proportions de caséines $\alpha_{S}$, le degré de minéralisation calcique des micelles et leur diamètre moyen, qui inter- viennent sur la cinétique de coagulation ou les caractéristiques rhéologiques du gel.

Coeff récup $M S=0,604$. (Caséines tot) $+0,175$. (Min ${ }^{\circ}$ calcique) - 0,397.(Caséines $\left.\alpha_{s}\right)-0,031$.(Diam micelles) + 44,7 $(R=0,83)$

Les influences de la teneur en caséines et de la minéralisation des micelles sont connues, et elles ont été observées par divers auteurs (eg Mocquot et al, 1954; Storry et al, 1983). La mise en évidence de l'intervention des proportions relatives des caséines, et plus particulièrement de l'effet négatif des caséines $\alpha_{s}$, est plus originale; il est possible que ce paramètre, qui est corrélé à la fermeté du gel, agisse, au moins en partie, par le canal de son influence sur les dimensions des micelles. L'équation [7] relative au rendement en matières azotées confirme l'incidence de la composition des caséines et l'effet favorable de l'accroissement de la proportion de caséine $\beta$.

Coeff récup $M A=0,762$. (Caséines tot) $+0,131$. (Ca ionisé) $+0,764$. (Caséine $\beta$ ) $+0,013 .(\mathrm{Pi}$ soluble $)+0,74(R=0,74)$

Tableau XII. Corrélations entre les paramètres de la coagulation par la présure. Correlations between renneting parameters.

\begin{tabular}{lccccc}
\hline & $\begin{array}{c}\text { Temps } \\
\text { de prise }\end{array}$ & $\begin{array}{c}\text { Vitesse de raff } \\
\text { du gel }\end{array}$ & $\begin{array}{r}\text { Fermeté } \\
\text { du gel }\end{array}$ & $\begin{array}{c}\text { Humid caillé } \\
\text { centr }\end{array}$ & $\begin{array}{c}\text { Poids sérum } \\
\text { égoutté }\end{array}$ \\
\hline $\begin{array}{l}\text { Vitesse de raff du gel } \\
\text { Fermeté du gel }\end{array}$ & $-0,44$ & 0,80 & & & \\
$\begin{array}{l}\text { Poids sérum égoutté } \\
\text { Coeff récup MS }\end{array}$ & 0,39 & 0,70 & 0,79 & $-0,63$ & $-0,50$ \\
Poids fines & 0,51 & & & $-0,51$ \\
\hline
\end{tabular}

NB : les coefficients de corrélation indiqués sont significativement différents de 0 au seuil $\alpha=5 \%$.

NB : the correlation coefficients indicated differ significantly from 0 at the threshold level of $\alpha=5 \%$. 
Compte tenu des corrélations ótroites qui existent entre le rendement fromager et le comportement du lait vis-à-vis de la présure (tableau XII), il était intéressant de préciser ces relations.

L'équation de régression reliant le coefficient de récupération de la matière sèche aux paramètres caractéristiques de la coagulation [8] confirme que la fermeté maximale du gel représente un critère d'appréciation des aptitudes fromagères des laits particulièrement significatif.

Coeff récup $\mathrm{MS}=0,075$. (Fermeté du gel) $+32,4(R=0,79)[8]$

\section{Relations entre les variables physico-chimiques et les paramètres caractéristiques des aptitudes fromagères en fabrication de pâte fraîche}

Le tableau XIII présente les corrélations significatives entre les variables physicochimiques et les paramètres caractéristiques des aptitudes fromagères en fabrication de pâte fraîche.
L'indice de viscosité est plus particulièrement influencé par la teneur en caséines et par certaines caractéristiques micellaires, l'hydratation et la minéralisation calcique notamment.

Quant au rendement en microfabrication de pâte fraîche, il apparaît être surtout influencé positivement par la teneur en caséines et le degré d'hydratation des micelles, et négativement par la minéralisation calcique de celles-ci.

L'analyse par régression multiple apporte certaines informations supplémentaires (équations [9]-[12] et tableau XI).

L'équation de régression de l'indice de viscosité des gels [9], confirme que cette caractéristique est avant tout déterminée par la teneur en caséines.

Indice viscosité $=0,116$. (Caséines tot) $+0,273(R=0,85)$

L'équation de régression de l'indice de comportement [10] montre que sa valeur augmente lorsque les concentrations en caséines et en citrate soluble diminuent et lorsque les valeurs du diamètre moyen et

Tableau XIII. Corrélations entre les variables physico-chimiques et les paramètres de la coagulation acide.

Correlations between physico-chemical variables and acid coagulation parameters.

\begin{tabular}{lccc}
\hline & $\begin{array}{c}\text { Indice } \\
\text { viscosité }\end{array}$ & $\begin{array}{c}\text { Indice } \\
\text { comportement }\end{array}$ & $\begin{array}{c}\text { Coeff } \\
\text { récup MS }\end{array}$ \\
\hline $\begin{array}{l}\text { Protéines } \\
\text { Caséines tot }\end{array}$ & 0,77 & & 0,85 \\
Caséine sol & 0,85 & & 0,88 \\
Variant $\beta$-lacto A & 0,57 & 0,42 & 0,43 \\
$\begin{array}{l}\text { Variant } \beta \text {-lacto B } \\
\text { Hydr micelles }\end{array}$ & & 0,34 & 0,36 \\
$\begin{array}{l}\text { Min calcique } \\
\text { Ca ionisé }\end{array}$ & 0,39 & $-0,34$ & 0,42 \\
Cit soluble & $-0,42$ & $-0,53$ & $-0,49$ \\
& & & \\
\hline
\end{tabular}

NB : les coefficients de corrélation indiqués sont significativement différents de 0 au seuil $\alpha=5 \%$.

NB : the correlation coefficients indicated differ significantly from 0 at the threshold level of $\alpha=5 \%$. 
du degré d'hydratation des micelles augmentent.

Indice comportement $=-0,051$. (Cit soluble) $+0,302$. (Hydr micelles) $+0,003$. (Diam micelles) - 0,011.(Caséines tot) $+0,353(R=0,75)$

L'équation de régression du coefficient de récupération de la matière sèche [11] fait apparaître deux paramètres physicochimiques particulièrement déterminants : la teneur en caséines et la proportion de caséine $\beta$.

Coeff récup $\mathrm{MS}=1,04$.(Caséines tot) $+0,251$. (Caséine $\beta)+5,15(R=0,90)$ [11]

Le rendement en matière sèche, exprimé en fonction des paramètres de l'aptitude à la coagulation acide, se traduit par l'équation [12] :

Coeff récup MS $=7,25$.(Indice viscosité) $+5,59$. (Indice comportement) + 13,4 $(R=0,86)$

À priori, cette équation peut surprendre dans la mesure où les indices de viscosité et de comportement interviennent avec le même signe, alors que ces 2 paramètres varient en sens inverse; sa validité a pu cependant être vérifiée sur des échantillons de laits individuels analysés dans les mêmes conditions que ceux qui ont servi à son calcul.

\section{CONCLUSION}

À partir d'échantillons de laits individuels, choisis selon un plan faisant intervenir plusieurs facteurs de variation, il a été procédé à la détermination des caractères physico-chimiques, de l'aptitude à la coagulation et du comportement en microfabrications fromagères.
Le traitement statistique des données a permis d'établir des liaisons entre variables et de sélectionner les paramètres physicochimiques dont l'influence sur les aptitudes fromagères sont les plus grandes.

Les laits étudiés présentent une grande variabilité de composition dans les teneurs en caséines, en azote non protéique, en calcium soluble, ainsi que dans les proportions relatives des caséines et les caractéristiques micellaires : degré d'hydratation et diamètre moyen. Cette variabilité, qui justifie a posteriori le plan d'échantillonnage, permet de bien mettre en évidence l'incidence des caractères physicochimiques sur le comportement technologique des laits.

Certaines relations entre les variables physico-chimiques ont pu être établies; s'il en est qui sont déjà bien connues (teneurs en protéines et en caséines, teneurs en caséines et en calcium colloïdal, proportions de caséine $\kappa$ et dimensions des micelles), d'autres sont moins évidentes. II en est ainsi, par exemple, de la liaison positive entre le pourcentage de caséines $\alpha_{s}$ et la dimension des micelles.

L'identification des variants génétiques des caséines a permis de mettre en évidence les relations associant le variant $B$ dela caséine $\kappa$ à l'accroissement de la proportion de caséine $\kappa$ et à la diminution du pourcentage des caséines $\alpha_{\mathrm{s}}$ ainsi qu'à celle du diamètre moyen des micelles.

La grande variabilité de composition se traduit par une variabilité également grande dans le comportement vis-à-vis de la présure, et des corrélations significatives sont observées entre variables physico-chimiques et technologiques. Parmi les plus intéressantes, on citera les liaisons positives entre la fermeté du gel et la teneur en caséines ou la proportion de caséine $\beta$, les corrrélations négatives entre cette variable rhéologique et la proportion de caséines $\alpha_{\mathrm{s}}$, ou la dimension des mi- 
celles. La vitesse de raffermissement, quant à elle, est corrélée négativement avec le pourcentage de caséines $\alpha_{s}$ et le diamètre moyen des micelles, et positivement avec la proportion de caséine $\kappa$.

On voit ainsi que parmi les paramètres qui déterminent l'aptitude du lait à la coagulation par la présure, figurent outre la teneur en caséines, les teneurs en calcium colloïdal et ionisé, ou la minéralisation des micelles (dont les influences favorables sont bien connues), la composition des caséines et le diamètre moyen des micelles. II apparaît clairement que l'accroissement de la proportion de caséines $\alpha_{\mathrm{s}}$ et celui de la dimension des micelles se traduisent par une moindre aptitude du lait à la coagulation par la présure, notamment par une réduction de la vitesse de raffermissement du gel et de sa fermeté maximale.

Le comportement des laits en microfabrication de pâte pressée présente lui aussi d'importantes variations et des corrélations assez étroites entre les critères de comportement fromager, et les caractères physico-chimiques des laits ont été mises en évidence. Ainsi, l'équation de régression [6] montre que parmi les paramètres ayant une influence marquante sur le rendement en matière sèche, on relève, à côté de la teneur en caséines évidemment déterminante, d'autres variables moins connues, telles la proportion de caséines $\alpha_{s}$, le diamètre moyen des micelles (corrélations négatives) et leur degré de minéralisation calcique (corrélation positive).

Par ailleurs, les relations observées entre les critères de rendement et les paramètres caractéristiques de l'aptitude à la coagulation par la présure méritent d'être soulignées et l'équation [8] permet une appréciation du coefficient de récupération de la matière sèche, avec un coefficient de détermination $\left(R^{2} \times 100\right)$ de $62,4 \%$, par une simple mesure de la fermeté du gel.
Des variabilités d'amplitude marquée ont été également observées dans les caractéristiques de la coagulation acide et le comportement des laits en microfabrication de pâte fraîche.

Les corrélations entre les paramètres de la coagulation acide et les variables physico-chimiques ainsi que l'équation de régression [11] montrent, là encore, qu'outre la teneur en caséines, la proportion relative de caséine $\beta$ exerce une influence positive sur le rendement de fabrication. En outre, l'existence d'une corrélation assez étroite entre les caractères rhéologiques du gel acide et le rendement rend possible une estimation indirecte de ce dernier, ce que confirme l'équation [12] $\left(R^{2} \times 100=72,2 \%\right)$.

Cette étude des relations entre les caractères physico-chimiques des laits et leur aptitude fromagère met ainsi en évidence :

- l'incidence de la composition des caséines, notamment du rapport caséines $\alpha_{s}$ ' caséine $\beta$; un rapport de valeur faible sera favorable pour la fermeté du gel présure et les rendements en fabrications de pâte pressée et de pâte fraîche.

- l'intérêt d'une prise en compte des caractéristiques de structure des micelles de caséines, notamment leurs dimensions et leur degré de minéralisation calcique; il apparaît, en effet, que ces 2 paramètres interviennent dans la détermination du comportement des laits vis-à-vis de la présure, des micelles de faible diamètre et d'un degré de minéralisation élevé sont à l'origine de gels plus fermes et de meilleurs coefficients de récupération de la matière sèche;

- certaines relations entre la nature des variants génétiques des protéines et la qualité technologique des laits; il est notamment confirmé que les laits contenant le variant $B$ de la caséine $\kappa$ présentent des 
aptitudes fromagères supérieures à ceux contenant la forme A de cette protéine.

Les prolongements technologiques de ces observations sont dans l'immédiat limités. Certes, il est possible d'améliorer les aptitudes fromagères de laits déficients par un enrichissement en caséines ou par ajout d'un sel de calcium assurant un accroissement de la teneur en calcium ionisé et de la minéralisation des micelles, mais ce sont là des corrections déjà connues et largement pratiquées. En revanche, on voit encore mal comment il serait possible d'intervenir par voie technologique sur les proportions relatives des caséines. C'est donc, semble-t-il, davantage par le canal de la sélection des animaux que l'on devrait pouvoir agir; celle-ci ne devrait sans doute plus être fondée sur la seule richesse en protéines ou en caséines, mais prendre également en compte la nature des variants et, dans la mesure où il s'agit de caractères liés au génotype, les proportions relatives des caséines et les dimensions des micelles.

Retenons, par ailleurs, que les corrélations établies entre certains paramètres caractéristiques de la coagulation et les rendements de fabrication rendent possible une estimation convenable des aptitudes fromagères des laits par la mise en œuvre de techniques d'exécution relativement simple et rapide, par exemple la mesure de la vitesse de raffermissement du gel présure et de sa fermeté maximale, ou celle de la viscosité du gel acide.

À la lumière de ces données, il reste certains points qui mériteraient une étude complémentaire :

- les formules d'établissement des rendements fromagers devraient être vérifiées sur des fabrications pilote, et si possible, en vraie grandeur;

- les rapports entre variants génétiques, composition des caséines, caractéristi- ques micellaires d'une part, et aptitudes fromagères de l'autre, devraient être précisés par la mise en œuvre de laits de petit mélange, laits de races différentes, typés sur la base de la nature des variants et des proportions respectives des fractions caséiniques.

\section{REMERCIEMENTS}

Ce travail s'inscrit dans le cadre d'un contrat entre l'INRA et l'Association pour le développement de la recherche dans l'industrie laitière (Arilait), avec une aide financière de la Direction générale de l'alimentation du ministère de l'Agriculture.

II a été réalisé avec la collaboration de $B$ Auberger et l'assistance technique de A Normand et M-T Le Tilly; leur concours a été déterminant et nous les en remercions.

Le dosage des caséines $\alpha_{s}, \beta$ et $\kappa$ a été effectué par la Station de recherche laitière INRA de Jouy-en-Josas, et l'identification des variants génétiques des protéines par le laboratoire de génétique biochimique INRA du même centre. Nous adressons nos remerciements à $M-F$ Mahé, G Miranda et JP Pélissier pour leur active coopération.

Nos remerciements vont également à G Staub et $P$ Le Tilly, de la Ferme expérimentale de I'INA-Paris-Grignon, qui ont facilité cette étude en permettant une bonne organisation des prélèvements d'échantillons.

\section{RÉFÉRENCES}

Alais C (1984) Science du lait, $4^{\theta}$ édn, Sepaic, Paris

Amram Y, Delespaul G, Vandeweghe J, Schneid N, Lenoir J (1982) Le refroidissement du lait et son comportement en fromagerie. Rev Lait Fr 404, 53-57

Banks JM, Muir DD (1984) Coagulum strength and cheese yield. Dairy Ind Int 49, 17-21, 36

Berridge NJ (1952) An improved method of observing the clotting of milk containing rennin. J Dairy Res 19, 328-329 
Brulé G, Lenoir J (1987) La coagulation du lait. In: Le Fromage, $2^{\ominus}$ édn (Eck A, ed) Lavoisier, Tec \& Doc, Paris

Buchberger J, Kiermeier F, Kirchmeier O, Graml $R$, Pirchner $F$ (1982) Effect of genetic variants of milk protein on milk composition. In: $X X I$ Int Dairy Congr (brief commun), Vol 1, Book 1, 40-41. Mir, Moscou

Burnett J, Scott Blair GW (1963) A speedcompensated torsiometer for measuring the setting of milk by rennet. Dairy Ind 28, 220223

Chahed S (1985) Rapports entre les dimensions des micelles de caséine, leur composition, et leur comportement vis-à-vis de la présure. Mémoire DEA Univ Caen, INA Paris-Grignon

Donnelly WJ, McNeill GP, Buchheim W, McGann TCA (1984) A comprehensive study of the relationship between size and protein composition in natural bovine casein micelles. Biochim Biophys Acta 789, 136-143

Ekstrand $B$, Larsson-Raznikiewicz $M$, Brännäng $E$, Swensson C (1981) Size distribution of casein micelles related to coagulation properties. Swed J Agric Res 11, 57-61

El-Negoumy AM (1972) Effects of polymorphic composition of calcium caseinate sols on their stability to rennin. $J$ Dairy Res $39,373-$ 379

Flueler O (1978) Neue Erkenntnisse über dir Labträgheit der Milch (1). Schweiz Milchwirtsch Forsch 7, 45-54

Ford GD, Grandison AS (1986) Effects of size of casein micelles on coagulation properties of skim milk. J Dairy Res 53, 129-133

Gervais A, Vermeire D (1983) A critical study and improvement of the cheese curd torsiometer. $J$ Texture Stud 14, 31-45

Grandison AS, Ford GD, Owen AJ, Millard D (1984a) Chemical composition and coagulating properties of renneted Friesian milk during the transition from winter rations to spring grazing. J Dairy Res 51, 69-78

Grandison AS, Ford GD, Millard D, Owen AJ (1984b) Chemical composition and coagulating properties of renneted milks from cows during early lactation. J Dairy Res $51,407-$ 416

Grandison AS, Ford GD, Millard D, Anderson M (1985) Interrelationships of chemical compo- sition and coagulating properties of renneted milks from dairy cows grazing ryegrass or white clover. J Dairy Res 52, 41-46

Grosclaude F (1988) Le polymorphisme génétique des principales lactoprotéines bovines. INRA, Prod Anim 1, 5-17

Guillou H, Miranda G, Pélissier JP (1987) Analyse quantitative des caséines dans le lait par chromatographie liquide rapide d'échange d'ions (FPLC). Lait 67, 135-148

Holt C, Muir D (1979) Inorganic constituents of milk. I. Correlation of soluble calcium with citrate in bovine milk. J Dairy Res 46, 433-439

Holt C, Parker TG, Dalgleish DG (1975) Measurement of particle size by elastic and quasielastic light scattering. Biochim Biophys Acta 400, 283-292

Jen JJ, Ashworth US (1970) Factors influencing the curd tension of rennet coagulated milk. Salt balance. J Dairy Sci 53, 1201-1206

Jenness R, Patton S (1959) Principles of dairy chemistry. J Wiley \& Sons Inc, New York

Kosikowski FV, Mocquot G (1958) Progrès de la technologie du fromage. FAO Rome

Lenoir J, Schneid N (1987) L'aptitude du lait à la coagulation par la présure. In: Le Fromage, $2^{\circ}$ edn (Eck A, ed) Lavoisier Tec \& Doc, Paris

Lombard SH (1982) Factors affecting enzymatic coagulation of milk and syneresis. In: Miles Annual Cheesemakers Symposium. Dep of Food Science, Univ Pretoria, South Africa

Losi G, Capella P, Castagnetti GB, Grazia L, Zambonelli C, Mariani P, Russo X (1973) Influenza delle varianti genetiche della caseina $\mathrm{K}$ sulla formazione e sulle caratteristiche della cagliata. Sci Technol Aliment 3, 373376

McLean DM, Graham ERB, Ponzoni RW, McKenzie HA (1982) Association between milk protein genotypes and milk yield and composition. In: XXI Int Dairy Congr (Brief commun), Vol 1, Book 1, 54-55, Mir, Moscou

McLean DM, Graham ERB, Ponzoni RW, McKenzie HA (1984) Effects of milk protein genetic variants on milk yield and composition. J Dairy Res 51, 531-546

McMahon DJ, Brown RJ, Richardson GH, Ernstrom CA (1984) Effects of calcium, phosphate, and bulk culture media on milk coagulation properties. J Dairy Sci 67, 930-938 
Mariani P, Losi G, Russo V, Castagnetti GB, Grazia L, Morini D, Fossa E (1976) Prove di caseificazione con latte caratterizzato dalle varianti $A$ e $B$ della $\kappa$-caseina nella produzione del formaggio Parmigiano-Reggiano. Sci Tec Latt-casearia 27, 208-227

Mariani P, Morini D, Losi G, Castagnetti GB, Fossa E, Russo V (1979) Ripartizione delle frazioni azotate del latte in vacche caratterizzate da genotipo diverso nel locus $\beta$ lattoglobulina. Sci Tec Latt-casearia 30, 153176

Marziali AS, Ng-Kwai-Hang KF (1986a) Relationships between milk protein polymorphisms and cheese yielding capacity. J Dairy Sci 69, 1193-1201

Marziali AS, Ng-Kwai-Hang KF (1986b). Effects of milk composition and genetic polymorphism on coagulation properties of milk. $J$ Dairy Sci 69, 1793-1798

Maubois JL, Mocquot G (1967) Comment ramener à la même teneur en substance sèche des fabrications de fromage en vue de comparer les «rendements" respectifs du lait en fromage. Rev Lait Fr 239, 15-18

Ménard JL, Tusseau S (1986) Incidence des variants génétiques de certaines protéines sur l'aptitude fromagère des laits. Étude ESA Angers, GIE Pays de Loire, ITEB

Mietton B (1986) La préparation des laits de fromagerie en technologie de pâtes molles. Ind Alim Agric 103, 951-963

Mocquot G, Alais C, Chevalier R (1954) Étude sur les défauts de coagulation du lait par la présure. Ann Technol Agric 3, 1-44

Ng-Kwai-Hang KF, Hayes JF, Moxley JE, Monardes HG (1984) Association of genetic variants of casein and milk serum proteins with milk, fat, and protein production by dairy cattle. J Dairy Sci 67, 835-840

Ng-Kwai-Hang KF, Hayes JF, Moxley JE, Monardes HG (1986) Relationships between milk protein polymorphisms and major milk constituents in Holstein-Friesian cows. J Dairy Sci 69, 22-26

Niki R, Arima S (1984) Effects of size of casein micelle on firmness of rennet curd. Jpn J Zootech Sci 55, 409-415

Okigbo LM, Richardson GH, Brown RJ, Ernstrom CA (1984) Estimation of syneresis, and cheese yield of bovine milk of different coagulation properties. J Dairy Sci 67 (suppl 1), 74

Okigbo LM, Richardson GH, Brown RJ, Ernstrom CA (1985a) Variation in coagulation properties of milk from individual cows. $J$ Dairy Sci 68, 822-828

Okigbo LM, Richardson GH, Brown RJ, Ernstrom CA (1985b) Casein composition of cow's milk of different chymosin coagulation properties. J Dairy Sci 68, 1887-1892

Okigbo LM, Richardson GH, Brown RJ, Ernstrom CA (1985c) Effects of pH, calcium chloride, and chymosin concentration on coagulation properties of abnormal and normal milk. J Dairy Sci 68, 2527-2533

Pearse MJ, Linklater PM, Hall RJ, Mackinlay AG (1986) Effects of casein micelle composition and casein dephosphorylation on coagulation and syneresis. J Dairy Res 53, 381-390

Peterson RF (1963) High resolution of milk proteins obtained by gel electrophoresis. J Dairy Sci 46, 1136-1139

Peterson RF, Kopfler FC (1966) Detection of new types of $\beta$ casein by polyacrylamide gel electrophoresis at acid $\mathrm{pH}$ : a proposed nomenclature. Biochem Biophys Res Commun $22,388-392$

Pierre A, Brulé G (1981) Mineral and protein equilibria between the colloidal and soluble phases of milk at low temperature. J Dairy Res 48, 417-428

Pierre A, Brulé G (1983) Dosage rapide du citrate dans l'ultrafiltrat de lait par complexation cuivrique. Lait $63,66-74$

Pyne GT, Mc Gann CA (1962) The influence of the colloidal phosphate of milk on the rennet coagulation. In: XVI Int Dairy Congr, Vol B. Andelbogtrykkeriet i Odense, Danemark, 611-616

Ramet JP, Weber F (1980) Contribution à l'étude de l'influence des facteurs de milieu sur la coagulation enzymatique du lait reconstitué. Lait 60, 1-13

Remeuf F, Lenoir J, Duby C (1989) Étude des relations entre les caractéristiques physicochimiques des laits de chèvre et leur aptitude à la coagulation par la présure. Lait $69,499-$ 518 
Rose D (1969) A proposed model of micelle structure in bovine milk. Dairy Sci Abstr 31, 171-175

Schaar J (1984) Effects of $\mathrm{K}$-casein genetic variants and lactation number on the renneting properties of individual milks. J Dairy Res 51, $397-406$

Schaar J (1985) Effects of genetic variants of $\kappa$ casein and $\beta$-lactoglobulin on cheesemaking. J Dairy Res 52, 429-437

Schmidt DG (1980) Colloidal aspects of casein. Neth Milk Dairy J 34, 42-64

Sood SM, Gaind DK, Dewan RK (1979) Correlation between micelle solvation and calcium content. N Z J Dairy Sci Technol 51, 18971902

Storry JE, Ford GD (1982) Some factors affecting the post clotting development of coagulum strength in renneted milk. J Dairy Res 49, 469-477

Storry JE, Grandison AS, Millard D, Owen AJ, Ford GD (1983) Chemical composition and coagulating properties of renneted milks from different breeds and species of ruminant. $J$ Dairy Res 50, 215-229

Swaisgood HE (1982) Chemistry of milk protein. In: Developments in Dairy Chemistry, Vol 1
(Fox PF, ed). Applied Science Publishers, London

Tervala H, Antila V, Syväjärvi J (1985) Factors affecting the renneting properties of milk. Meijeritiet Aikak 43, 16-25

Thompson MP, Boswell RT, Martin V, Jenness R, Kiddy CA (1969) Casein-pellet solvation and heat stability of individual cows milk. J Dairy Sci 52, 796-799

Tomassone R (1987) Comment interpréter les résultats d'une régression linéaire. ITCF, Paris

Tomassone R, Lesquoy E, Millier C (1983) La régression : nouveaux regards sur une ancienne méthode statistique. Masson-INRA, Paris

Waagner-Nielsen WE, Berntsen G, Hansen S, Larsen BV, Edelsten D (1982) Rennet coagulation of casein micelles of different size. In: $X X I$ Int Dairy Congr (brief commun, vol 1, book 2, 257-258. Mir Publ, Moscou

Wake RG, Baldwin RL (1961) Analysis of casein fractions by zone electrophoresis in concentrated urea. Biochim Biophys Acta 47, 225239

Yun S, Ohmiya K, Shimizu S (1982) Role of $\beta$ casein in milk curdling. Agric Biol Chem 46, 443-449 